\title{
DESIGN AND APPLICATION OF A SINGLE-BEAM GAMMA DENSITOMETER FOR VOID FRACTION MEASUREMENT IN A SMALL DIAMETER STAINLESS STEEL PIPE IN A CRITICAL FLOW CONDITION
}

\author{
HYUN-SIK PARK" and CHANG-HWAN CHUNG \\ Thermal Hydraulics Safety Research Center, Korea Atomic Energy Research Institute, \\ 1045 Daedeokdaero, Yuseong, Daejeon, 305-353, Korea \\ "Corresponding author. E-mail : hspark@kaeri.re.kr
}

Received November 15, 2006

Accepted for Publication May 29, 2007

A single-beam gamma densitometer is utilized to measure the average void fraction in a small diameter stainless steel pipe under critical flow conditions. A typical design of a single-beam gamma densitometer is composed of a sealed gammaray source, a collimator, a scintillation detector, and a data acquisition system that includes an amplifier and a single channel analyzer. It is operated in the count mode and can be calibrated with a test pipe and various types of phantoms made of polyethylene. A good average void fraction is obtained for a small diameter pipe with various flow regimes of the core, annular, stratified, and bubbly flows. Several factors influencing the performance of the gamma densitometer are examined, including the distance between the source and the detector, the measuring time, and the ambient temperature. The void fraction is measured during an adiabatic downward two-phase critical flow in a vertical pipe. The test pipe has an inner diameter of $10.9 \mathrm{~mm}$ and a thickness of $3.2 \mathrm{~mm}$. The average void fraction was reasonably measured for a two-phase critical flow in the presence of nitrogen gas.

KEYWORDS : Gamma-Densitometer, Void Fraction, Non-Condensable Gas, Two-Phase Flow, Critical Flow

\section{INTRODUCTION}

The void fraction is one of the most important parameters in a gas-liquid two-phase critical flow conditions. A great deal of effort has been devoted to discovering methods to measure it accurately. The void fraction in a gas-liquid flow is currently measured using a variety of techniques including volumetric, electrical, optical, ultrasonic and radiation methods. Among these methods, radiation methods are most commonly used in many applications as they are simple and non-intrusive. Radiation methods include the use of fast neutron scattering and attenuation techniques as well as the use of gamma and X-ray attenuation techniques. Compared with other radiation techniques, the gamma ray technique is well developed and more versatile because gamma rays of different energies can be chosen depending on the test section used.

Chan and Banerjee [1] suggested a design procedure for a single-beam gamma densitometer and designed two densitometers for refilling and rewetting experiments and flow boiling experiments, which are both transient experiments. Good average void measurements were obtained for relatively fast transients. Jiang and Rezkallah [2] performed an experimental study of the suitability of using a gamma densitometer for void fraction measurements in a gas-liquid flow in a small diameter tube. They found that the performance of the gamma densitometer is good during adiabatic upward and downward two-phase flows. Chu and Song [3] applied the gamma ray attenuation technique to measure the void fraction of a steam-water mixture flowing downward in a downcomer annulus in a direct vessel-injection experiment. The design parameters of previous gamma densitometers are listed in Table 1.

In the present study the void fraction of a two-phase flow with a critical velocity is measured in a small-diameter vertical tube using a single-beam gamma densitometer. The two-phase critical flow experiments [4] were performed at KAERI to simulate a SMART pipe break accident [5]. SMART is designed to produce fresh water from seawater while producing electricity as well. The influence of a non-condensable gas on the critical discharge rate is an important safety issue, and accurate void fraction measurements are a major concern. As the test section is operated in high-pressure and high-velocity conditions, it consists 
Table 1. Design Parameters of Previous Gamma Densitometers

\begin{tabular}{|c|c|c|c|c|}
\hline Design Parameter & Chan, et al. (\#1) [1] & Chan, et al. (\#2) [1] & Jiang, et al.[2] & Chu, et al.[3] \\
\hline \multicolumn{5}{|l|}{ Test Section } \\
\hline Type & Tube & Tube & Tube & Annulus \\
\hline Material & Zircaloy-2 & Quartz & Acryl & Stainless steel \\
\hline Outer Diameter & $19.05 \mathrm{~mm}$ & $13.0 \mathrm{~mm}$ & $11.11 \mathrm{~mm}$ & $22.0 \mathrm{~mm}$ (outer wall) \\
\hline Thickness & $0.864 \mathrm{~mm}$ & $1.15 \mathrm{~mm}$ & $0.7925 \mathrm{~mm}$ & $10.0 \mathrm{~mm}$ (inner wall) \\
\hline \multicolumn{5}{|l|}{ Radiation Source } \\
\hline Isotope & Cobalt-57 & Plutonium-238 & Cesium-137 & Cobalt-60 \\
\hline Energy & $122,136.5 \mathrm{keV}$ & $11.6 \sim 21.7 \mathrm{keV}$ & $662 \mathrm{keV}$ & $1173,1333 \mathrm{keV}$ \\
\hline Emission ratio & $85.2,11.1 \%$ & $\sim 13 \%$ & $85.1 \%$ & $99.86,99.98 \%$ \\
\hline Sensitivity & $\sim 0.24$ & $\sim 1.0$ & $\sim 0.007$ & NA \\
\hline Statistical Error & $<5 \%$ & $<5 \%$ & NA & $<5 \%$ \\
\hline Count Rate & $\sim 500000 \mathrm{cps}$ & $\sim 30000 \mathrm{cps}$ & $\sim 50000 \mathrm{cps}$ & $\sim 57000 \mathrm{cps}$ \\
\hline \multicolumn{5}{|l|}{ Scintillation Detector } \\
\hline Type & $10 \%$ lead loaded plastic & $\operatorname{NaI}(\mathrm{Tl})$ & $\operatorname{NaI}(\mathrm{Tl})$ & $\operatorname{NaI}(\mathrm{Tl})$ \\
\hline Efficiency & $\sim 50 \%$ & $100 \%$ & $100 \%$ & $100 \%$ \\
\hline \multicolumn{5}{|l|}{ Radiation Shielding } \\
\hline Material & Steel & Steel & NA & Lead \\
\hline Thickness & $>38.1 \mathrm{~mm}$ & $>9.5 \mathrm{~mm}$ & NA & $>120 \mathrm{~mm}$ \\
\hline
\end{tabular}

of thick-walled stainless steel. Taken as a whole, these findings show that the gamma ray attenuation technique is the most reliable method for measuring the void fraction inside a stainless steel pipe with a thick wall.

\section{DESIGN OF A SINGLE-BEAM GAMMA DENSITOMETER}

The major components of a single-beam gamma densitometer are shown in Fig. 1.

The gamma densitometer consists of a sealed gamma-ray source, shielding material, a radiation detector, a signal processing unit and a traversing system. The selection of each of these components depends on the geometry and the material of the test section over which the void fraction is to be measured. The test section in the present study consists of stainless steel with an inner diameter of $10.9 \mathrm{~mm}$ and an outer diameter of $17.3 \mathrm{~mm}$. Fig. 2 shows a picture of an installed single-beam gamma densitometer that includes its sealed source, a collimator, a detector, and a traversing system. The design specifications of each component are explained by Park et al. [6] in detail.

\subsection{Design Principle}

The gamma-ray attenuation technique is based on the experimentally shown fact that the intensity of a gamma beam decreases exponentially as it passes through matter. When the gamma densitometer is operated in count mode, the beam intensity can be represented by the counting rate, which is obtained by using a scintillation detector and a counter. The average void fraction in a gas-liquid two-phase flow condition can be derived as

$$
\alpha=\frac{\ln \left(C R_{0} / C R_{\alpha}\right)}{\ln \left(C R_{0} / C R_{1}\right)}
$$

where $C R_{0}, C R_{1}$, and $C R_{a}$ are the count rates of a zero void, unit void, and the void to be measured, respectively.

The water transmission ratio is defined as the ratio of 


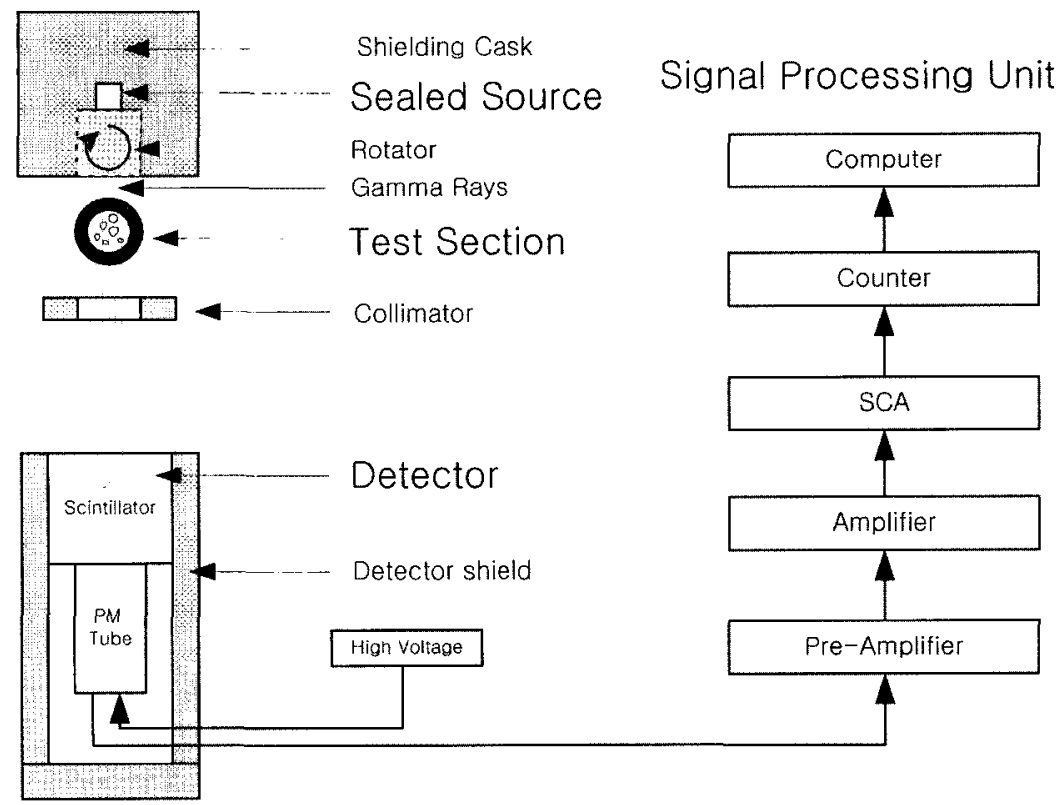

Fig. 1. Schematic Diagram of a Single-beam Gamma Densitometer

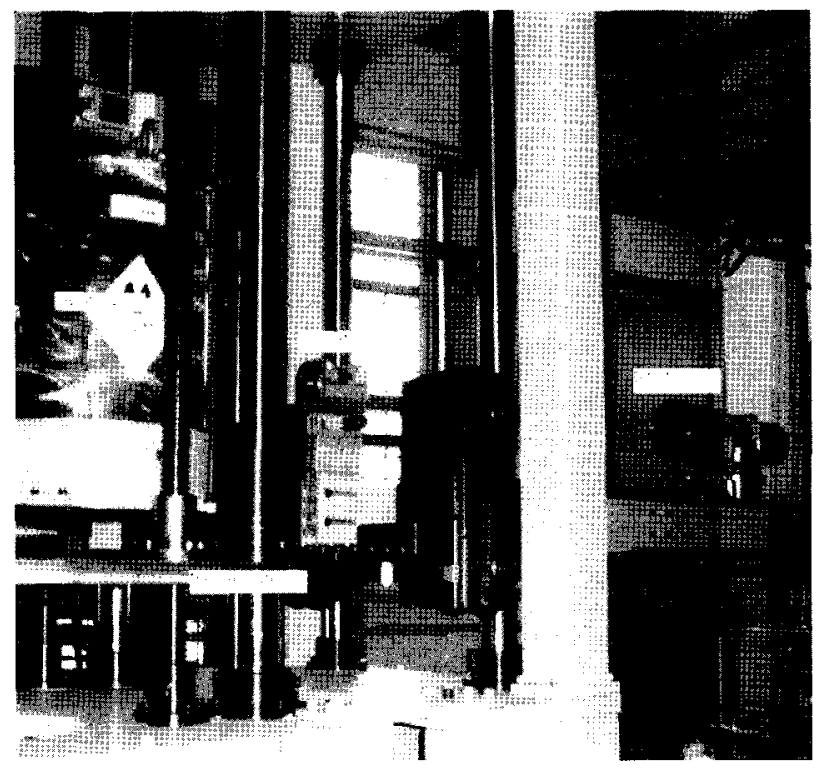

Fig. 2. A Picture of the Installed Single-beam Gamma Densitometer

the transmitted fluxes when the test pipe is full and when it is empty. For a given test pipe, the transmission of the gamma beam through the walls and the water content in the pipe can be calculated independently. A high water transmission ratio is advisable in the design of a gamma densitometer. When choosing the appropriate radiation source in this application, the level of sensitivity to the water content and the emission ratio of the desired gammas are the major concerns. A high sensitivity and high emission ratio are desirable.

The statistical error of the count measurement for a single-beam gamma densitometer operating in count mode is the most important parameter in the calculation of the required source strength. The derived statistical error is inversely proportional to the sensitivity and the square root of the total count number.

$$
\varepsilon_{\alpha}=\frac{1}{S \sqrt{N_{\alpha}}}
$$

Here, $N_{a}$ is the number of photons detected by the counting system during a certain period and $S$ is the sensitivity of the gamma beam to the water content in the test section:

$$
S=\frac{N_{1}-N_{0}}{\left(N_{1}+N_{0}\right) / 2}=2 \frac{(1-T R)}{(1+T R)}
$$


where $T R$ is the transmission ratio $\left(T R=N_{0} / N_{1}\right)$. The sensitivity of the single-beam gamma densitometer is approximately 0.05 for the present test section.

The use of a lower energy gamma is desirable because a higher sensitivity will result in a smaller error for a given count rate. The count rate required for a given statistical error is determined by Eq. (2) and the total measuring time. The required source strength can be estimated from the calculated count rate by considering several factors, including the counting period, beam size, collimation distance, scintillation efficiency and the emission ratio of the gamma ray source. The source is contained in a shielding container. The shielding container provides an adequate amount of shielding, and the gamma beam can be turned on and off using an appropriate shutter system.

The design procedure of Chan and Banerjee [1] was applied to design the present gamma densitometer. After the geometry and material of the test section are specified, the radioactive isotope and its photon energy are chosen, and the required source strength is calculated. The shielding requirements are then estimated and the radiation detector and the counting system are chosen according to the source strength.

\subsection{Radiation Source}

The source should have mono-energetic gamma photons with energies high enough to penetrate the walls of the test section with a measurable attenuation but low enough to be sensitive to the water content. In terms of sensitivity, lower energy gammas are desirable. However, if the photon energy is too low, the beam will be heavily attenuated by the test section and thus more intense sources will be necessary to compensate for the high level of attenuation. On the other hand, a higher energy beam can penetrate the walls with less attenuation, but it may be extremely insensitive to the water content. Therefore, a compromise has to be made in choosing the most suitable source.

In the present experiment, Cobalt- 60 was chosen as it has the highest emission ratio for the desired gammas and a relatively long half-life. The emission ratios of Cobalt60 are 99.86 and $99.98 \%$, respectively, for the principal photon energies of 1173 and $1333 \mathrm{keV}$, which are very high when compared with other radiation sources. Cobalt60 was obtained from HANARO at KAERI. It was found that a source activity of approximately $30 \mathrm{mCi}$ is sufficient to limit the statistical error for the void fraction measurement to within 0.02 , which can be calculated from Eq. (2). The maximum count rate of the gamma densitometer was determined to be within the count rate limit of $\mathrm{NaI}(\mathrm{Tl})$.

\subsection{Equipment for a Radiation Shielding}

As mentioned above, Cobalt- 60 was used as a gamma ray source and was shielded properly in a lead cask after a shielding calculation was performed using the MCNP code. The radiation source was cylindrically shaped and sealed in a shielded container made of lead, as the gamma energy involved was very high. The minimum thickness used was $15 \mathrm{~cm}$. The total dose received by both the researcher and the public were well below the maximum permissible annual dose rates due to the design of the container. The radiation source was fixed in the center of the container and the radiation beam could be turned on or off using a rotating cylinder with a collimating hole along the direction of the beam.

\subsection{Radiation Detector and Signal Processing Unit}

The signal processing system was chosen according to the count rates required. When choosing the scintillator, a high level of detection efficiency is desirable and a short decay constant is necessary for high count rates in order to avoid saturation while the system is operating in count mode. Among the many available scintillators, $\mathrm{NaI}(\mathrm{Tl})$ is most commonly used due to its high detection efficiency. However, its count rate is limited to within 100,000 counts per second due to its relatively long decay time.

Individual gamma pulses were generated by a gamma radiation from the scintillator through a photo-multiplier, were amplified by a preamplifier, and were further amplified and shaped by an amplifier/TSCA. The signal from an amplifier/TSCA was analyzed by a multi-channel analyzer or counted by a counter. The signal from the multi-channel analyzer was analyzed by an exclusive analyzing program. The accumulated counter signal was transmitted to a PC by a RS-232C communication.

\section{CALIBRATION OF A GAMMA-DENSITOMETER}

The gamma densitometer was calibrated with various methods after the output signals from the detector, preamplifier, and amplifier/TSCA were ascertained to be sound using an oscilloscope. The gamma densitometer was calibrated using a test pipe equipped with polyethylene phantoms, which simulate gas-liquid twophase flow regimes in a vertical tube. Additionally, a change of the count rate was investigated using a test pipe and varying the distance between the source and the detector, the measuring time and the ambient temperature.

The energy spectrum was obtained using a multichannel analyzer, as shown in Fig. 3. In the gamma densitometer, the signals from the lower energy region were rejected using a single channel analyzer. The count rates, which are inversely proportional to the amount of water, were obtained to provide the void fraction. The energy spectra in Fig. 3 show that two characteristic peaks were well captured by this device and that the signals are sound. 


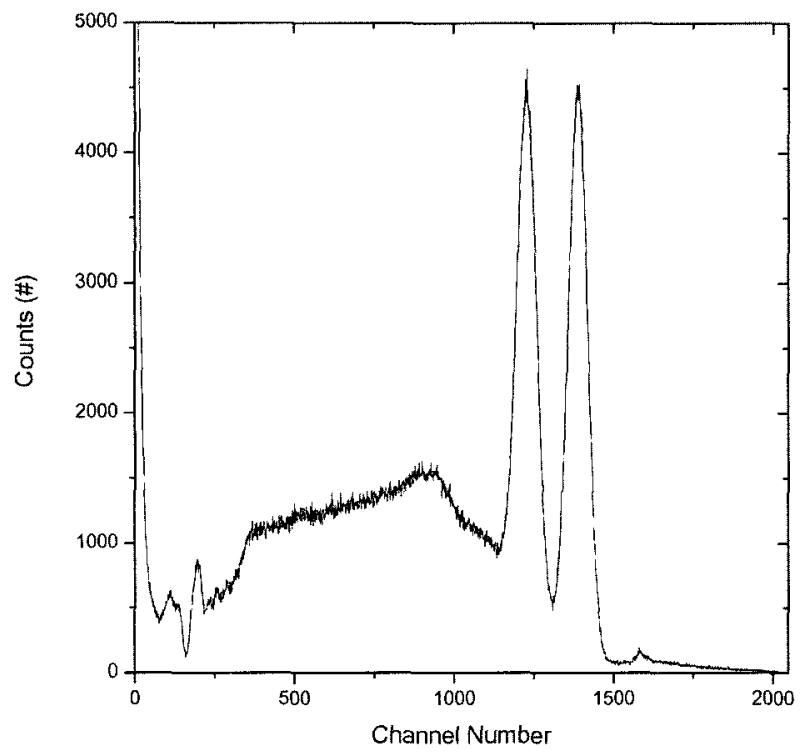

Fig. 3. Energy Spectra of the Cobalt-60 Using a NaI(Tl) Crystal

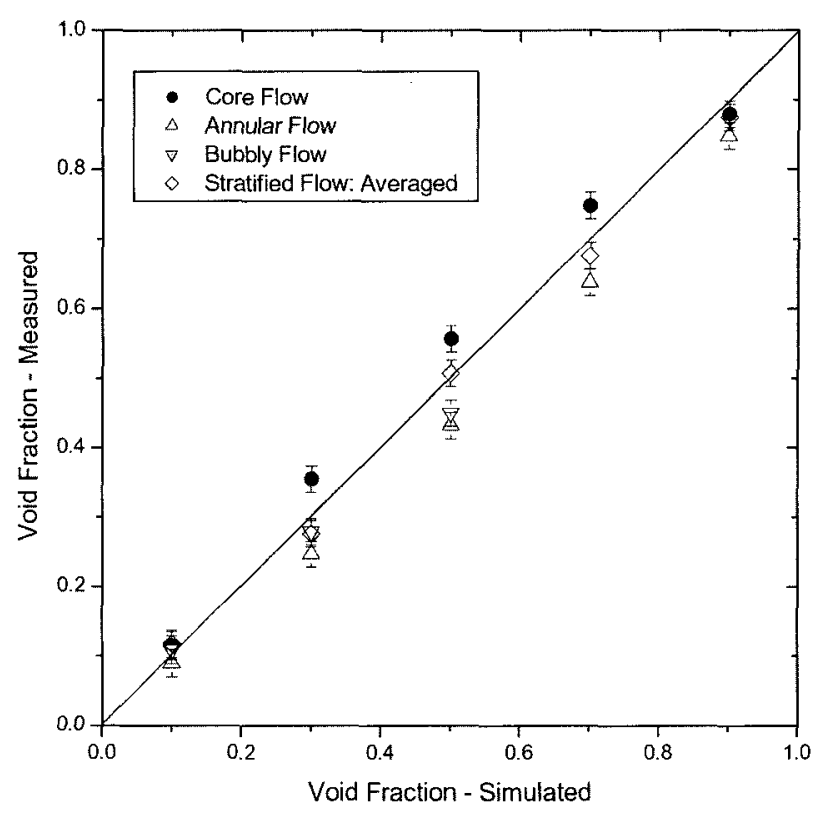

Fig. 4. Comparison of the Void Fractions Measured by a Gamma Densitometer with those Simulated by Polyethylene Phantoms

the degree of attenuation varies as the amount of the material is changed. However, several parameters can influence the performance of the instrumentation. Its response can be affected by factors such as the distance between the source and the detector, the measuring time, and the ambient temperature.

Fig. 5 shows the results of several tests conducted to investigate the influence of the distance between the detector and the source. The distance between the source and the detector varied between 0.582 and $2.582 \mathrm{~m}$. During these tests, the ambient temperatures were between 9.5 and $11.9^{\circ} \mathrm{C}$ and all measuring times were 50 seconds. As expected, the results show that the count rate decreases exponentially as the distance between the source and the detector increases. Another series of experiments were performed to examine the influence of changing the measuring time.

Fig. 6 shows the measured time against the counting rate. The measuring times varied between 0.05 and 500 seconds. During these tests, the ambient temperatures were between 10.3 and $10.9^{\circ} \mathrm{C}$ and the distance between the source and the detector was $1.582 \mathrm{~m}$. The counting rate was nearly constant for all experiments, which shows that good measurements with a two-phase flow are possible. In addition, the dependency of the count rate on the ambient temperature was examined. The ambient temperatures varied between 3.3 and $8.2^{\circ} \mathrm{C}$. 


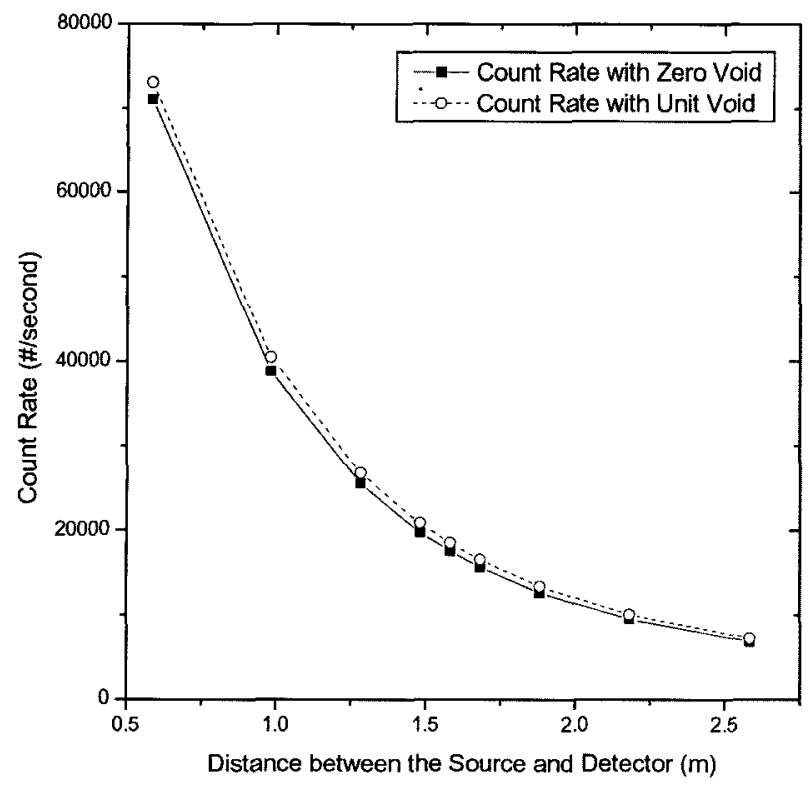

Fig. 5. Change of the Count rate with Variations of the Distance Between the Source and the Detector

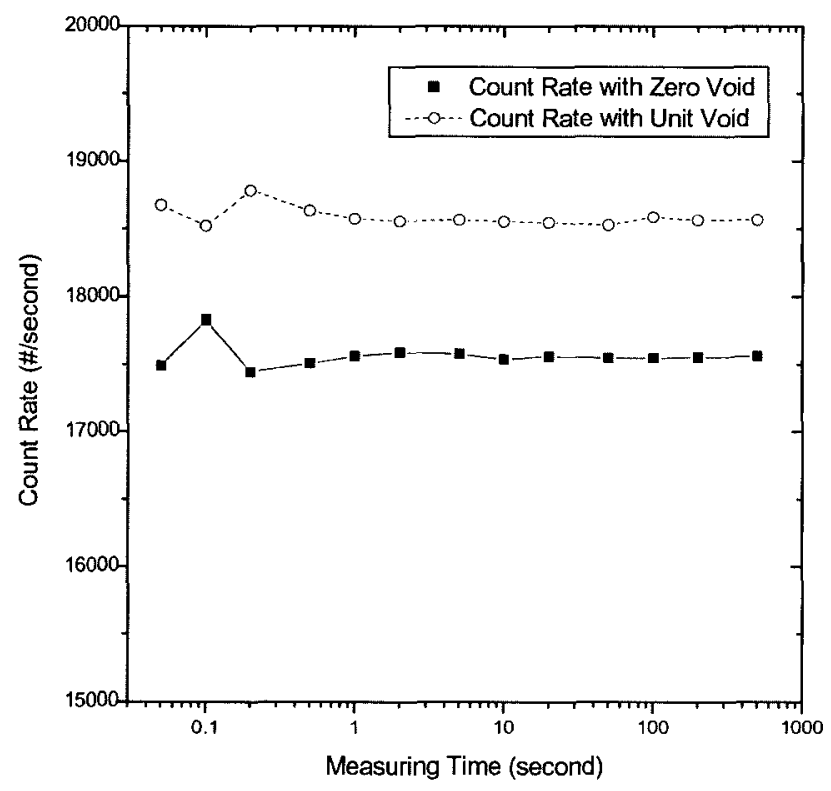

Fig. 6. Change of the Count rate with Variations of the Measuring Time

During these tests, the measuring times were 50 seconds and the distance between the source and the detector was $1.604 \mathrm{~m}$.

As shown in Fig. 7 , the count rate was nearly constant with the variation of the ambient temperature.

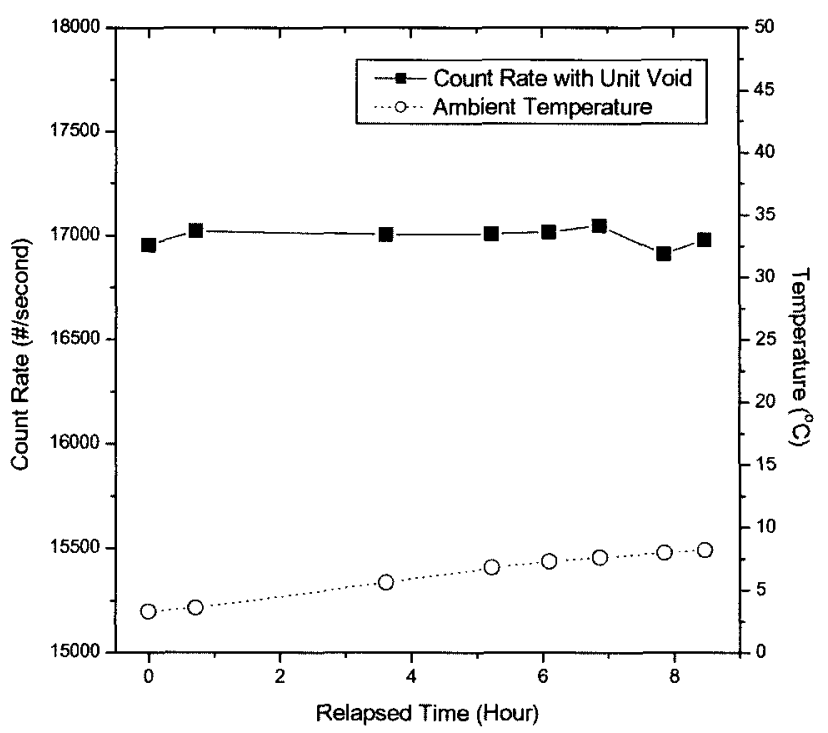

Fig. 7. Change of the Count rate with Variations of the Ambient Temperature

This shows that the gamma densitometer was not affected by a change of the ambient temperature during the tests.

\section{VOID FRACTION MEASUREMENT USING A GAMMA DENSITOMETER}

The void fraction of the gas-liquid flow with the critical velocity was measured in a small-diameter vertical tube using a gamma densitometer. The two-phase critical flow test facility, as employed in the previous experiment [4], was used. Its test section was replaced by a new one in order to measure the void fraction using a single-beam gamma densitometer.

\subsection{Two-Phase Critical Flow Test Facility and T2GDM}

An experimental work [4] on a two-phase critical flow was performed at KAERI to ascertain the effect of a non-condensable gas, nitrogen gas in this case, in a critical flow under high-pressure conditions. The test facility is shown in Fig. 8. The major components of the test facility are the pressure vessel, the test section, the suppression tank, and the nitrogen gas supply system. The test section, termed T2, is a sharp-edged stainless steel pipe with an inner diameter of $10.9 \mathrm{~mm}$, a thickness of $3.2 \mathrm{~mm}$, and a length of $1000 \mathrm{~mm}$. It is instrumented with five thermocouples and eighteen pressure taps to measure the distributions of the temperature and pressure. In this 


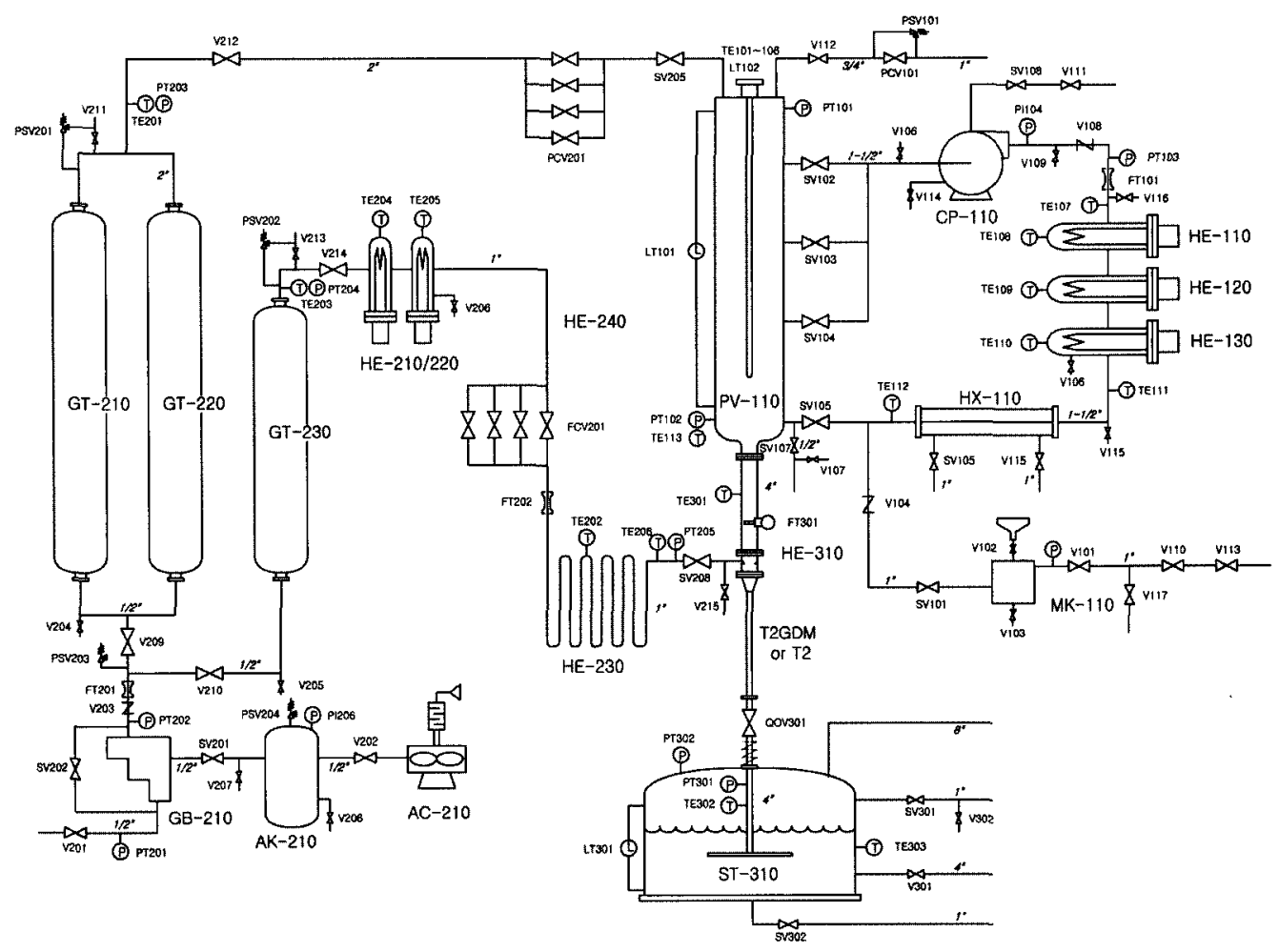

Fig. 8. Schematic of the Two-Phase Critical Flow Test Facility at KAERI

Table 2. Test Matrix for the Void Fraction Measurement

\begin{tabular}{c|c|c|c}
\hline Stagnation Pressure(bar) & Injected Nitrogen Gas Flow Rate(kg/s) & Measured Water Flow Rate(kg/s) & Initial Void Fraction \\
\hline 40 & $0 \sim 0.100$ & $2.267 \sim 4.987$ & $0 \sim 0.4111$ \\
70 & $0 \sim 0.105$ & $4.128 \sim 6.830$ & $0 \sim 0.2179$ \\
\hline
\end{tabular}

facility, water is discharged from a pressure vessel, nitrogen gas is injected into the test loop and mixed with water, and the mixture flows in the test section with a critical velocity. In the test, the stagnation temperature and pressure of the coolant were measured in the pressure vessel, and the coolant was discharged through the discharge pipe. Its flow rate was measured upstream of the test section, and the temperature and the flow rate of the injected nitrogen gas through the injection nozzle were measured. The total flow rate is the sum of the water and air flow rates. The maximum measurement errors for the water and air flow rates are 0.5 and $2.0 \%$ of the full range, respectively.

A twin test section to measure the void fraction was designed and the single-beam gamma densitometer was applied. T2GDM, inside which the void fraction was measured using the gamma densitometer with a traverse system, was newly installed in the existing two-phase critical flow test facility. The test section was a stainless steel pipe with the same length and inner diameter as T2.

\subsection{Test Matrix and Experimental Procedure}

10 runs of experiments were performed to measure the void fraction. Table 2 shows the test matrix for the void fraction measurement.

The experimental procedure is as follows:

1) The pressure vessel was filled with water to a predetermined level.

2) The nitrogen gas was injected into the pressure vessel to provide the stagnation pressure.

3) The pressure was kept constant using the pressure control valve connected to the nitrogen supply tank.

4) The water was discharged from the pressure vessel. 
5) The nitrogen gas was injected into the discharged water.

6) The steady-state data of the critical flow rate, the pressure, the temperature and the void fraction were acquired.

\subsection{Results and Discussion}

A set of experiments was performed using T2GDM to acquire the experimental data of the critical flow rate and the void fraction. The stagnation temperature of the pressure vessel was at a normal atmospheric temperature and its stagnation pressures were 40 and 70 bars. In each case, the water was discharged from the pressure vessel without any gas injection in the early stage. After the flow was stabilized, the nitrogen gas was injected into the discharged water in the following stage.

The volumetric averaged void fraction upstream of the test section was calculated using the flow rates of the discharged water and the injected nitrogen gas as measured by separate flow meters, and the void fraction was estimated using the empirical correlation of Yamazaki [7]. The void fraction in the test section was also measured using the single-beam gamma densitometer. In the present tests, the measuring time was between 10.4 and 29.6 seconds and the distance between the source and the detector was optimized to provide a maximum count rate. Fig. 9 shows a comparison of the void fractions measured by the gamma densitometer with those calculated from the volumetric averaged void fraction. The measured void fractions at the center of the test section are always higher than those

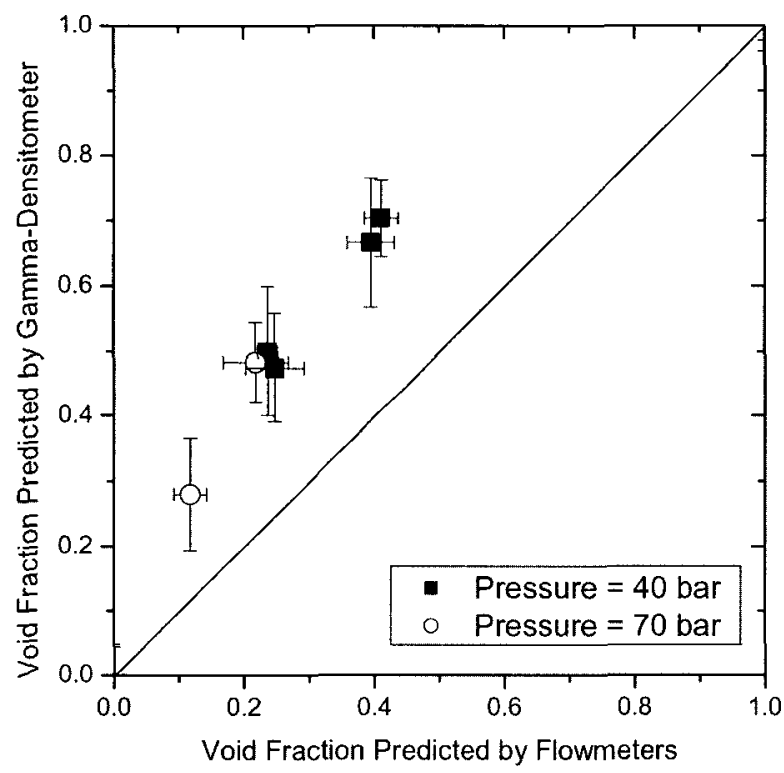

Fig. 9. Comparison of the Void Fractions Measured Using a Gamma Densitometer with those Calculated from the Volumetric Averaged Void Fraction at the inlet of the test section.

There are two explanations for this. The first is related to the test section, which has a smaller inner diameter $(10.9 \mathrm{~mm})$ compared to that of the connecting pipe. Mishima and Ishii [8] showed that the probability of coalescence in a small-diameter pipe is much higher compared to a tube with a larger diameter; this was also observed by Jiang and Rezkallah [2]. The second explanation is related to the fluid in the test section, which is in a critical flow condition. In two-phase critical flow conditions, the pressure is decreasing along the test section and an additional void is generated near the outlet of the test section to increase the void fraction.

The nitrogen gas was injected into the mixing part of the vertical pipe, which is located $0.8 \mathrm{~m}$ upstream of the test section. The measurement results show that the void fraction is increased while the two-phase mixture passes through the test section. A certain amount of void may be generated in the test section before the two-phase mixture arrives at the outlet of the test section in a critical flow condition.

Numerical simulations were performed using the MARS 2.1 code [9] to validate the variation tendency of the void fraction. The MARS code used for this analysis is a best-estimate computer code, and it adopts the Henry-Fauske critical flow model to calculate the critical flow rate as a default option.

Fig. 10 shows the typical void fraction distribution in a critical flow test of T2GDM-70-C, which was performed at a stagnation pressure of 70 bar with a noncondensable

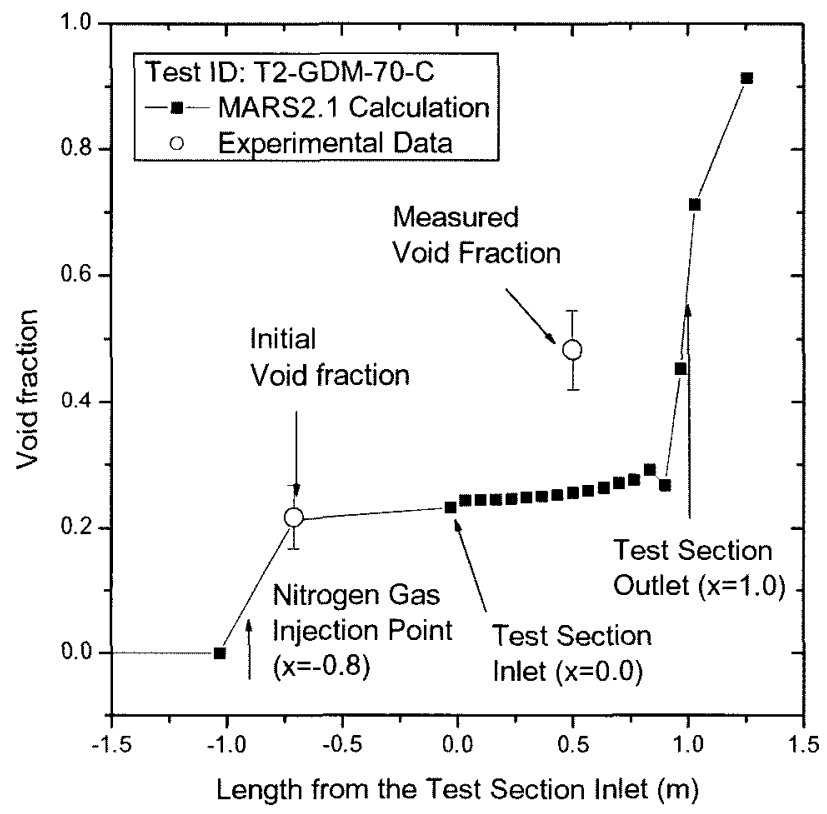

Fig. 10. Typical Void Fraction Distribution in a Critical Flow Test Facility 
gas present. The simulation result shows that the void fraction at the outlet is similar to that measured by the gamma densitometer.

Fig. 11 shows a comparison of the void fractions measured using the gamma densitometer with those predicted at the outlet by the MARS code. In the MARS calculation, the void fraction was acquired at the last node of the test section. In the present calculation, 15 nodes were used to simulate the test section and the center of the last node was at $0.033 \mathrm{~m}$ upstream of the test section. A sensitivity calculation shows that the void fraction at the last node of the test section varies very little as the node number increases when the node number is greater than 10. The comparison results show good agreement to within a standard deviation of 9.2 and $9.4 \%$ for stagnation pressures of 40 and 70 bars, respectively. More detailed experimental data regarding the axial void fraction distribution in the test section appears to be necessary.

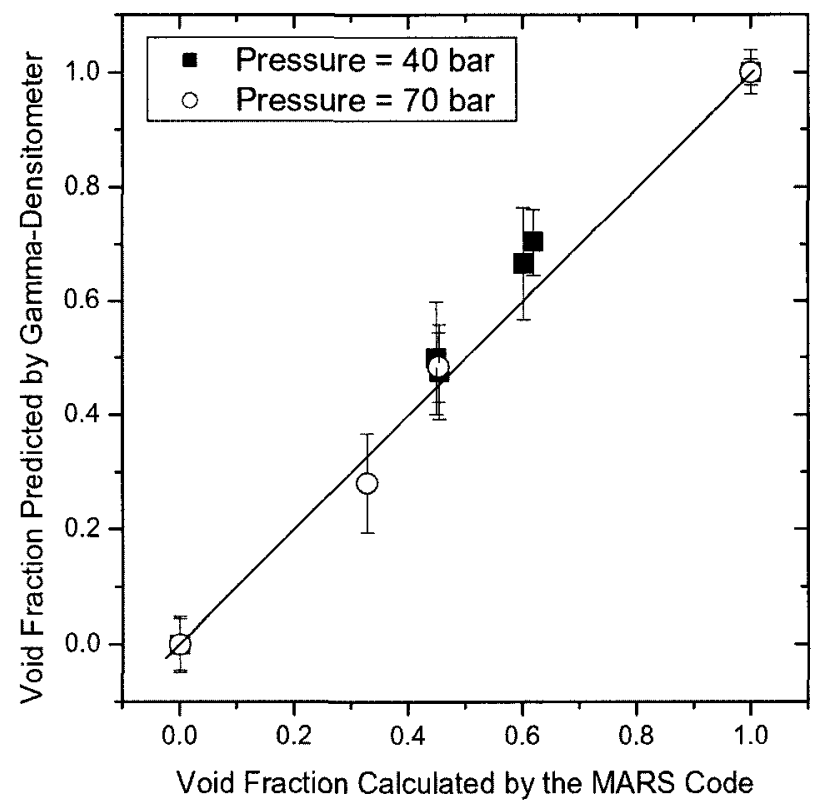

Fig. 11. Comparison of the Void Fractions Measured Using a Gamma Densitometer with those Predicted by the MARS Code

\section{CONCLUSIONS}

A single-beam gamma densitometer operated in count mode was developed and applied to measure the crosssectional average void fraction in a vertical tube with a small diameter under two-phase critical flow conditions. Its design is simple and compact, and it can be applied to a small-diameter pipe with an inner diameter of $10.9 \mathrm{~mm}$ and a relatively thick wall in high-pressure and high-velocity conditions. The single-beam gamma densitometer consists of a sealed gamma ray source, shielding material, a radiation detector, a signal processing unit and a traversing system. The gamma densitometer is calibrated with a range of methods, and its design was shown to work well. The performance of the densitometer was examined using phantom pieces intended to simulate different void fractions and flow distributions.

The void fraction of a flowing fluid with a critical velocity was measured using the two-phase critical flow test facility with the test section of T2GDM. Test results show that the gamma attenuation technique provides a reasonable average void fraction in a tube with a small diameter. The void fraction was measured at a high pressure using the single-beam gamma densitometer, and the measurement results show that the void fraction increases when the mixture passes through the test section under two-phase critical flow conditions.

\section{NOMENCLATURE}

CR Count rate $[\# / \mathrm{s}]$

$N \quad$ Number of photons [\#]

$S \quad$ The sensitivity [-]

TR Transmission ratio [-]

(Greek)

$\alpha \quad$ Void fraction [-]

$\varepsilon \quad$ Statistical error [-]

\section{(Subscripts)}

$0 \quad$ Zero void fraction

1 Unit void fraction

$\alpha \quad$ Void fraction to be measured

\section{ACKNOWLEDGEMENTS}

This work was performed under the National Mid- \& Long-Term Nuclear R\&D Program sponsored by the Ministry of Science and Technology (MOST) of Korea.

\section{REFERENCES}

[1] A. M. C. Chan and S. Banerjee, "Design Aspects of Gamma Densitometers for Void Fraction Measurements in Small Scale Two-Phase Flows," Nuclear Instruments and Methods, 190, 135 (1981).

[2] Y. Jiang and K. S. Rezkallah, "An Experimental Study of the Suitability of Using a Gamma Densitometer for Void Fraction Measurements in Gas-Liquid Flow in a Small Diameter Tube," Meas. Sci. Technol., 4, 496 (1993).

[3] I. C. Chu and C. H. Song, "Development and Performance Evaluation of 32-Channel Gamma Densitometer for the Measurement of Flow Pattern and Void Fraction in the Downcomer of MIDAS Test Facility," KAERI/TR2045/2002, KAERI (2002).

[4] S. K. Chang, H. S. Park, and C. H. Chung, "Analysis of 
the Test Results for the Two-Phase Critical Flow with Non-Condensable Gas," KAERI/TR-2242/2002, KAERI (2002).

[ 5 ] M. H. Chang, J. W. Yeo, S. Q. Zee, et al., "Basic Design Report of SMART," KAERI/TR-1242/2002, KAERI (2002).

[6] H. S. Park, C. H. Chung, S. K. Chang, et al., "Measurement of Void Fraction in a Vertical Tube under Critical Flow Conditions using a Single-Beam Gamma Densitometer," KAERI/TR-2306/2002, KAERI (2002).
[7] Y. Yamazaki and K. Yamaguchi, "Characteristics of Cocurrent Two-Phase Downflow in Tubes - Flow Pattern, Void Fraction and Pressure Drop," J. Nul. Sci. Technol., 16, 245 (1979).

[8] Mishima, K. and Ishii, M. "Flow Regime Transition Criteria for Upward Two-Phase Flow in Vertical Tubes," Int. J. Heat Mass Transfer, 27, 723 (1984).

[9] W. J. Lee, B. D. Chung, J. J. Jeong, et al., "Improved Features of MARS 1.4 and Verification," KAERI/TR1386/99, KAERI (1999). 